\title{
Numerical Study of the Formation and Drying Kinetics of A Capillary Bridge of Trehalose solution between two Parallel Hydrophilic Fibres
}

\author{
Yejiong Yu, Aidong Yang, Hua Ye, Julian F Dye, Zhanfeng Cui* \\ Institute of Biomedical Engineering, Department of Engineering Science \\ University of Oxford \\ *Correspondence to: Professor Zhanfeng Cui, Department of Engineering Science, University of \\ Oxford. Email: zhanfeng.cui@eng.ox.ac.uk
}

\begin{abstract}
Formation and vitrification of the capillary bridges is believed critical to matrix assisted room temperature drying of biologics. In this paper, a Capillary Bridge number ( $C B$ number) is derived to predict the wetting morphology with $\mathrm{CB}>0$ to form capillary bridges, otherwise, form droplets. To simulate the drying of the formed capillary bridge with pure water or trehalose solution, a continuum model is constructed to predict its surface instability and solute distribution. For the evaporation of a pure water capillary bridge, the numerical simulation results demonstrate that liquid is driven by capillary pressure from the central area to the sides, which eventually triggers the rupture. During the drying of trehalose solution-loaded capillary bridges, Marangoni flow overcomes the capillary flow and pushes liquid towards the centre instead, resulting in solidification except for the very dilute solutions. The results improve understanding and design of matrix assisted room temperature drying of biotherapeutics.
\end{abstract}

Keywords: Matrix assisted room temperature (MART) drying, Capillary bridge, Liquid bridge, Evaporation, Marangoni flow, Capillary Bridge Number

\section{Introduction}

Matrix assisted room temperature (MART) drying uses three dimensional porous matrices to load the solution of biological therapeutics supplemented with trehalose and removes water at ambient temperature and pressure. MART drying can save significant fraction of energy and drying time compared to freeze drying. MART drying proved to be effective to preserve some vaccines for long term storage ${ }^{1-}$ 3 .

The mechanism of how biologics and their functions are preserved during MART drying is not clear. Electron-microscopic study indicated vaccine particles need to be trapped within the vitrified sugar to preserve their functions ${ }^{1-6}$.
Apart from providing possible hydrogen bonding replacement to water, trehalose helps to form and stabilize capillary bridges during drying. The aim of this paper is to develop mathematical models to predict formation of capillary bridges and their stability during drying.

The capillary bridge, also called the liquid bridge, is a volume of liquid spanning two or more solid surfaces by surface tension and capillary pressure ${ }^{7-9}$, typically recognized in granular flows ${ }^{10,11}$, microfluidic dynamics ${ }^{12,13}$, oil recovery ${ }^{14}$ and pattern formation of solidified materials ${ }^{15}$. Since Young studied the surface tension of the capillary bridge in $1805^{16}$, much work has been done to investigate the shape and stability of capillary bridges between surfaces with differences in wettability, rugosity 
and geometry, principally focusing on spherical particles ${ }^{17-25}$. Less attention has been paid to the capillary bridge in fibre matrices and the majority of these studies have analysed the wetting morphologies experimentally ${ }^{8,26-29}$. Boulogen et $\mathrm{al}^{30}$ presented experimental results on the evaluation of the viscous liquid evaporation at the cross point of two nylon fibres aligned with different angles (column, drop and mixed) and compared the evaporation rate with a simplified predication.

Capillary bridge structure in fibre matrices determines the wetting, the degree of liquid retention and the drying rate ${ }^{26,31}$. The bridge geometry is a principle parameter in the drying dynamic defining either the break point or solidification point. Several experiments have studied the rupture of a pure water capillary bridge due to the evaporation ${ }^{32-35}$. However, the drying kinetics of a solution capillary bridge, such as a sugar solution, is still poorly understood. In this paper, we seek to model these two processes.

(a)

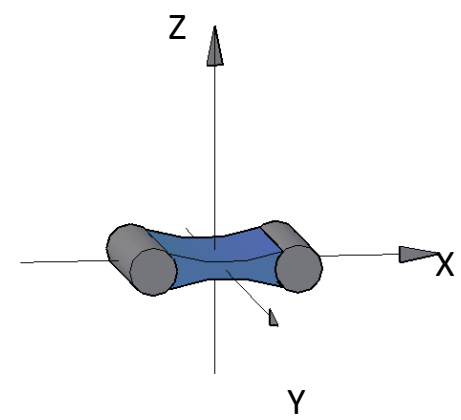

(b)

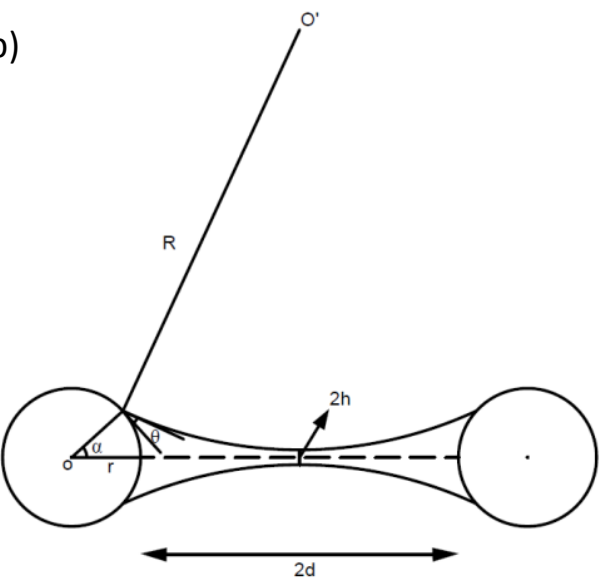

Figure 1: The three-dimensional diagram of capillary bridge between two hydrophilic fibres (a) and the cross-sectional illustration of capillary bridge for detailed analysis.

To develop a mathematical model of capillary bridge drying in three dimensional fibre matrices presents a complex problem, due to the loss of axisymmetric structure ${ }^{29,36}$. Herein, the problem is simplified by considering the capillary bridge on two parallel hydrophilic fibres. The axial symmetry of capillary bridge along Y-axis (Figure 1(a)) and the absence of gravity reduces the difficulty of the geometry determination and drying dynamic simulation $^{37,38}$.

For a submillimetre system, the Bond number $(B o)$ describing the ratio of gravitational force against surface tension, is less than 1, indicating that gravitational force may be neglected ${ }^{39,40}$. Therefore, the shape of the capillary bridge is governed by liquid surface tension, which is expressed by the YoungLaplace Equation (Eq.1) (0-43, $^{\text {, }}$

$$
\sigma \kappa=\sigma\left(\frac{1}{R_{1}}+\frac{1}{R_{2}}\right)=\Delta p,
$$

where $\sigma$ is surface tension of the loaded solution and $\Delta p$ is capillary pressure. $\kappa$ is the curvature and $R_{1} \& R_{2}$ are the two principle radii of curvature. In this case, the principle radius in $Y$ direction is infinite, $R_{1}=\infty . R_{2}$ is the principle radius of the curvature for the middle point at gas-liquid interface, shown as $R$ in Figure $1(\mathrm{~b})$. The curvature $(\kappa)$ of capillary bridge is expressed as $\kappa=\frac{1}{R}+\frac{1}{\infty}=\frac{1}{R}$, which is constant along the surface. Constant curvature, namely nodoid geometry, makes the surface a perfect arc.

To our knowledge, theoretical prediction of capillary bridge formation and modelling drying kinetics of the capillary bridge have not been described previously, despite the established theories of the geometry and wetting morphology ${ }^{27,28}$. Therefore, this study starts with a geometric analysis to find the criterion for capillary bridge formation between two 
paralleled fibres. Then, a mathematical model is constructed to explore the flow within the capillary bridge driven by evaporation of either pure water or sugar solution. Notably, the varying physical properties of a solution with different initial concentration increases the complexity of the mathematical model.

\section{Geometric Criterion for Forming Capillary Bridge}

A cross-sectional diagram of capillary bridge formed between two parallel hydrophilic fibres with mathematical parameters was constructed (Figure 1(b)) based on previous work ${ }^{37,44}$, where $d$ is the halved separation distance between two fibres, $\alpha$ represents the half-filling angle, $\theta$ stands for contact angle of liquid and solid surface, $H$ is the thickness of capillary bridge neck. The two equations Eq. 2 and Eq. 3 were established in the literature:

$$
\begin{gathered}
\frac{H}{2}=h=r \sin \alpha-[R-R \sin (\alpha+\theta)] \\
\frac{R}{r}=\frac{1+\frac{d}{r}-\cos \alpha}{\cos (\alpha+\theta)}
\end{gathered}
$$

However, the criterion for capillary bridge formation, the maximum distance between two fibres which could sustain capillary bridge, has not been previously derived.

A capillary bridge is assumed to be formed between two fibres if the half thickness of capillary bridge neck $(h)$ greater than zero. Namely, the result of Eq. 2 must be greater than zero with given fibre properties. However, the half-filling angle cannot be directly measured but may be substituted by four other measurable parameters (Eq. 3). Rearranging the equation above gives the value of half-filling angle $(\alpha)$ based on the liquid and solution properties (Eq. 4), which must be located between zero and $\mathrm{pi} / 2$ :

$$
\alpha=\arcsin \left(\frac{-(r+d)}{\sqrt{r^{2}+2 R r \cdot \cos \theta+R^{2}}}\right)
$$

$$
+\operatorname{acrtan}\left(\frac{\frac{\mathrm{r}}{\mathrm{R}}+\cos \theta}{\sin \theta}\right)
$$

Eq. 4

Substituting the half-filling angle into the Eq. 2, the criterion of forming capillary bridge, $h>0$, is simplified as

$$
C B=2 R r \cdot \cos \theta-d^{2}-2 r d>0 .
$$

Here, we call this criterion the Capillary Bridge number ( $C B$ number). If the $C B$ number is greater than zero, the fibre matrix can maintain a capillary bridge structure, based on the geometry of fibre and liquid properties. If the CB number is less than zero, only a droplet conformation of liquid spread could exist.

The maximum distance between parallel fibres with given properties that could sustain a capillary bridge is deduced as follows:

$$
D_{\text {max }}=2 d_{\text {max }}=2 \sqrt{r^{2}+2 R r \cos (\theta)}-2 r
$$

The main challenge in determining Capillary bridge number is to find a value of the princle radius of the curvature $(R)$, which is related to the Laplace pressure through Youngs-Laplace equation. The Kelvin equation(Eq. 7) gives a relationship between Laplace pressure and relative humidity $(R H)$.

$$
K T \ln (R H)=V_{m} \Delta P
$$

where $\mathrm{K}$ is Boltzmann constant, $\mathrm{T}$ is the temperature in Kelvin unit, $\mathrm{RH}$ is relative humidty, $V_{m}$ is molecular volume of liquid. Combining those two equation, the Kelvin radius is defined as

$$
R_{k}(\text { Kevin radius })=\frac{\sigma \cdot V m}{K T \ln (R H)}
$$

Eq. 8. At $20^{\circ} \mathrm{C}$, the value of $\left(\sigma \cdot V_{m}\right) / K T$ is $0.54 n m$ for pure water with surface tension of $72.8 \mathrm{mN} /$ $m^{45,46}$. Although the Kelvin radius has been applied to calculated as a principle radius of curvature, this is only relevant in the nanometre range. In larger scale, observed values are 
several orders of magnitude greater than kelvin radius $^{47-49}$.

The principal radius could be calculated if the unit volume $(V)$ of the capillary bridge is obtained. Combining Eq.4 with Eq.9 to eliminate the half-filling angle, the relationship between principle radius $(R)$ and unit volume $(V)$ with other three measurable parameters $(r, d, \theta)$ can be found. The function expressed this relationship is too long to be shown here.

$$
\begin{aligned}
V=4 r \sin \alpha \cdot( & r-r \cos \alpha+d) \\
& -R^{2}[\pi-2(\alpha+\theta)]-2 \alpha r^{2} \\
& +4 R^{2} \sin (\alpha+\theta) \cdot \cos (\alpha+\theta)
\end{aligned}
$$

\section{Modelling of Drying Dynamics of Capillary Bridge}

A mathematical model for capillary bridge drying with either pure water or solution has been made based on previous works involving droplet drying simulations ${ }^{50,51}$. A disaccharide, trehalose, regarded as surfactant is used in the system. It is widely used as cryoprotectant ${ }^{52-54}$.

The mathematical formulations of drying the capillary bridge with pure water overlaps with the same model for a sugar solution. Therefore, only the mathematical framework of evaporating capillary bridge with trehalose is presented here.

\subsection{Mathematical framework and assumptions}

A Cartesian coordinate system (Figure 2) is applied to simulate the drying process in the capillary bridge structure, in which $x$ and $z$ symbolize the horizontal and vertical coordinates. Since the gravitional force is ignored, the geometry of capillary bridge is symmetric in both $\mathrm{x}$ and $\mathrm{z}$ directions. For simplicity, the top-right corner of the capillary bridge is taken to represent the whole capillary bridge for detailed analysis (Figure 2(b)).

There are several assumptions are made to ease the calculations. The mixture is regarded as an incompressible, Newtonian liquid and the solute was considered as evenly distributed over the capillary bridge at starting point. During desiccation, dry air is assumed to flow with constant velocity at certain relative humidity and constant temperature. The density of the solution is regarded as constant throughout desiccation ${ }^{51,54,55}$.

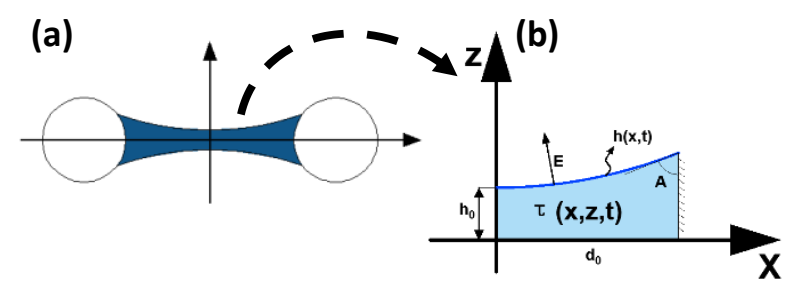

Figure 2: Schematic diagram of the Capillary bridge model (a) and mathematical model of capillary bridge for simulation (b).

The contact angle between liquid and fibre surface does not change with solution concentration $^{56}$. The right-side and bottom of capillary bridge was simplified as a straight and rigid wall. Therefore, the dynamics of contact line during evaporation is not included in this work.

\subsection{Governing equations}

Since the mixture is regarded as incompressible, Newtonian liquid, the continuity and momentum conservation equation in bulk mixture are expressed as:

$$
\begin{gathered}
(\nabla \cdot \boldsymbol{U})=0 \\
\rho \boldsymbol{U}_{t}+\rho(\boldsymbol{U} \cdot \nabla) \boldsymbol{U}=-\nabla \boldsymbol{p}+\mu(\tau) \nabla^{2} \boldsymbol{U}
\end{gathered}
$$

where the subscripts denote the derivative. $\nabla$ is the differential operator. $\boldsymbol{U}$ is the velocity vector in the system, which has two components $(u, w)$ in $\mathrm{x}$ and $\mathrm{z}$ directions. $\rho$ and $\boldsymbol{P}$ stand for liquid density and pressure, respectively.

Viscosity $(\mu)$ of the mixture is dependent on the concentration $(\tau)$ of trehalose measured in $\mathrm{mol} \cdot L^{-1}$. An empirical equation of the viscosity 
and concentration of trehalose was described previously ${ }^{57}$ :

$$
\mu(\tau)=A_{0} e^{\mathrm{A}_{1} \psi}
$$

Eq. 12

where,

$$
\psi(\text { weight precentage })=\frac{\tau \cdot M_{T r e}}{\rho}
$$

The empirical parameters $A_{0}$ and $A_{1}$ are relevant to the temperatures. $M_{\text {Tre }}$ is the molar mass of trehalose. Experimentally determined values of $A_{0}$ and $A_{1}$ were $1.2932 \times 10^{-3}$ and 0.0471 respectively at $9^{\circ} \mathrm{C}($ Appendix 2 ), the wetbulb temperature for drying at $25^{\circ} \mathrm{C}$ with $5 \%$ relative humidity.

Trehalose is a non-volatile and non-reducing solute $^{58}$, whose mass is conserved during desiccation. The concentration is governed by a standard convective-diffusion equation in the bulk solution:

$$
\frac{\partial \tau}{\partial t}+\nabla \boldsymbol{U} \tau=D(\tau) \nabla^{2} \tau
$$

Eq. 13

where $D$ refers to the diffusion coefficient of the solute in the units of $m^{2} \cdot s^{-1}$, which is also subject to the concentration ${ }^{59}$ :

$$
D(\tau)=\frac{5 \cdot 10^{-8}}{e^{-13} \cdot \psi^{1.1}} \cdot e^{\frac{-1500 \cdot e^{1.9 \psi}}{T}}
$$

Eq. 14

where $T$ represents the temperature in Kelvin.

\subsection{Boundary conditions}

To resolve the governing equations above, several boundary conditions are set. At the substrate $(z=0)$, no solute flux is assumed due to symmetry ${ }^{60,61}$. In addition, a no-slip condition is applied for simplicity, rather than moving boundary condition.

$$
\begin{gathered}
{\frac{\partial \tau}{\partial z_{\mid z=0}}}^{=}=0 \\
\boldsymbol{U}_{\mid z=0}=0
\end{gathered}
$$

Eq. 15

Eq. 16

The solute accumulated at the interface due to drying diffuses back to the bulk solution (Eq. 17). The kinematic boundary conditions (Eq.
18), associated with evaporation rate are involved:

$$
\tau\left(\boldsymbol{U}-\boldsymbol{U}_{s}\right) \cdot \boldsymbol{n}-D(\tau) \nabla \tau \cdot \boldsymbol{n}=0,
$$

$$
E=\left(\boldsymbol{U}-\boldsymbol{U}_{s}\right) \cdot \boldsymbol{n}
$$

Eq. 18

where $\boldsymbol{U}_{\boldsymbol{s}}$ is the interfacial velocity and $\boldsymbol{n}$ is the unit normal vector along the interface. $E$ refers to the evaporation rate, which will be discussed later.

The extension and rearrangement of the interfacial kinematic boundary condition (Eq.19) shows the relationship between the changing rate of surface height with time $\left(h_{t}\right)$ and horizontal velocity component $(u)$ :

$$
E=\frac{1}{\kappa}\left(w-u h_{x}-\aleph \cdot h_{t}\right)
$$

where,

$$
\aleph=\sqrt{1+h_{x}^{2}}
$$

In the meantime, the stresses in normal and tangential directions are balanced at the interface, where involves the capillary flow (Eq.20) and Marangoni flows (Eq.21). Capillary flow is driven by capillary pressure, which is related with surface tension $(\sigma)$ and curvature (к) of the interface.

At the tangential direction of interface, Marangoni effect is considered in this paper, which pull the liquid from low surface tension region to high surface tension region. Therefore, the tangential stress should be balanced by the difference of the surface tension along the interface, the right-hand side of Eq.21.

Since the scale of bulk properties in the gas phase is much smaller than those of liquid, the system can be viewed as a one-sided model. In the one-sided model, the descriptions of the normal and tangential stress balance are presented as:

$$
(\boldsymbol{T} \cdot \boldsymbol{n}) \cdot \boldsymbol{n}=p_{\text {cap }}=\sigma \cdot \kappa=\frac{\sigma \cdot h_{x x}}{\left(\sqrt{1+h_{x}^{2}}\right)^{3}}
$$

Eq. 20 
Eq. 21

where $\boldsymbol{T}$ is the stress tensor and $\nabla_{S}$ is surface gradient operator. $\boldsymbol{n}$ and $\boldsymbol{t}$ are the unit normal and tangential vector along the interface respectively.

Surface tension of the solution is involved in both normal and tangential stresses, which is dependent with temperature and solute concentration. As the system temperature assumed to be constant, the solute concentration becomes the determining factor. For trehalose, surface tension increases with trehalose concentration and the relationship is illustrated by the surface tension coefficient $\left(\gamma_{\text {tre }}\right)^{61}$ :

$$
\gamma_{t r e}=\frac{\partial \sigma}{\partial \tau}=0.411 \mathrm{dyn} / \mathrm{cm}
$$

\subsection{Dimensionless scaling}

After scaling and simplification by using the lubrication approximation $\left(\epsilon=\frac{h_{0}}{d_{0}} \ll 1\right)$, the governing equations for surface height (Eq. 22) and trehalose concentration (Eq. 23) are expressed as follows. The detail formulations are shown in Appendix $A$.

$$
\begin{gathered}
\frac{\partial \check{h}}{\partial \check{t}}=-\frac{h_{0} U_{0}}{d_{0}} E-\frac{\partial(\check{h} \bar{u})}{\partial \check{x}} \\
\frac{\partial \check{\tau}_{0}}{\partial t}=-\bar{u} \cdot \check{\tau}_{0 \check{x}}+\frac{\check{D}}{P e \cdot \check{h}}\left(\check{h} \check{\tau}_{0_{x}}\right)_{x}+\frac{\check{\tau}_{0}}{h U_{0} \epsilon} \cdot{ }^{22} \\
\bar{u}=\frac{1}{\check{h}(\check{x})} \int_{0}^{\check{h}(\check{x})} \check{\breve{u} d \check{z}}
\end{gathered}
$$

Marangoni Effect

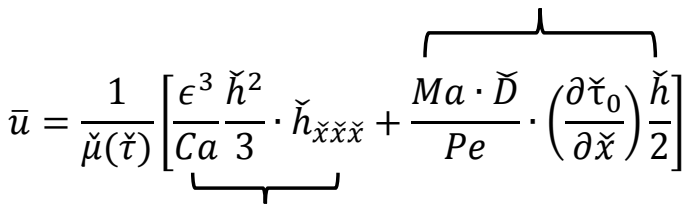

$$
\begin{aligned}
& \text { Capillary Effect }
\end{aligned}
$$

Eq. 24 where the tildes denote the dimensionless variables. $\frac{\partial \breve{h}}{\partial \check{t}}$ illustrates the changing rate of scaled height with the respect of scaled time. $E$ is the evaporation rate, $h_{0}, U_{0}$ and $d_{0}$ are the dimensional scalings of height, velocity and distance respectively. $\widetilde{D}$ and $\breve{\mu}$ are the scaled diffusion coefficient and viscosity, both of which are related to concentration. $\mathrm{Ca}$ and $\mathrm{Ma}$ represent capillary number and Marangoni number respectively. Peclet number $(\mathrm{Pe}=$ $\left.\frac{U_{0} d_{0}}{D}\right)$ is a parameter to evaluate the dominance of convective versus diffusive transportation during the desiccation.

Surface evolution (Eq. 22) demonstrates the relationship between the convection flux and evaporation rate. In terms of concentration variation with time, Eq. 23 shows that the concentration is related to convection flux, diffusion flux and solute accumulation by evaporation.

Both Eq.22 and Eq.23 cannot be resolved without knowing the average velocity cross $z$ direction $(\bar{u})$. From the boundary conditions of surface stress balances, the velocity is illustrated by the Capillary Effect and the Marangoni effect (Eq.24). As mentioned above, Capillary Effect is raised by the surface curvature while Marangoni Effect is raised by the surface tension differences. These equations and relationships are fully explained in Appendix A.

\subsection{Evaporation rate}

The evaporation rate is the mass transfer rate of water across the gas-liquid interface, with units of $\left(m \cdot s^{-1}\right)$. The gas is assumed to obey the ideal gas law.

$$
\begin{aligned}
E_{i}= & \frac{M_{w} \cdot N_{A}}{\rho}=\frac{M_{w} \cdot k_{c}\left(c_{s}-c_{\infty}\right)}{\rho} \\
& =\frac{M_{w} \cdot k_{c}}{\rho}\left(\frac{p_{s}(\tau)}{R T_{s}}-\frac{p_{\infty}}{R T_{\infty}}\right)
\end{aligned}
$$

Eq. 25

where $k_{c}$ is mass transfer coefficient. $p_{w}, p_{\infty}$ are the partial pressure at the interface and in the gas phase respectively. 
The ambient temperature $\left(T_{\infty}\right)$ is $25^{\circ} \mathrm{C}$, with $5 \%$ relative humidity. According to the psychometric chart the wet-bulb temperature $\left(T_{s}\right)$ at the liquid surface is $9^{\circ} \mathrm{C}$. The partial vapour pressure of water in gas phase $\left(p_{\infty}\right)$ and the ambient temperature are assumed as constant all the time. The partial vapour pressure at the interface $\left(p_{s}\right)$ is dependent on trehalose concentration and the relationship complies with Raoult's Law.

\section{Simulated Results of Drying Dynamics and Discussion}

\subsection{Determination of capillary shape}

As mentioned above, the initial shape of the interface is an arc. Therefore, the surface profile along the $x$ direction is expressed in dimensionless terms as:

$$
\breve{h}(\check{x}, \check{t}=0)=\frac{R}{h_{0}}+1-\frac{1}{h_{0}} \sqrt{R^{2}-d_{0}{ }^{2} \check{x}^{2}}
$$

where the contact angle $(\theta)$ of liquid on fibre was set to $\pi / 6$ and the distance of two fibres (D) was $1 \mu \mathrm{m}$. The diameter of fibre is $3 \mu \mathrm{m}$. The principle radius of curvature was set to 0.7 $\mu m$ as arbitrary values representative of a typically system.

The variables following are all scaled except for trehalose weight percentage. The distance is scaled by the half distance between two fibres $\left(\check{x}=x / d_{0}\right)$, where $\breve{x}=0$ means the centre of the capillary bridge and $\check{x}=1$ stands for the end of the capillary bridge. The height is scaled by the half of the initial neck thinness $\left(\check{h}=h / h_{0}\right)$.

\subsection{Simulation results with pure water}

Since the structure at the centre $(x=0)$ is symmetric, there is no flux across the symmetry line, which means the horizontal velocity and the derivative of concentration with the respect of $x$ at the centre were both zero.

$$
\breve{u}(\check{x}=0, \check{t})=\bar{u}(\check{x}=0, \check{t})=0
$$

(a)

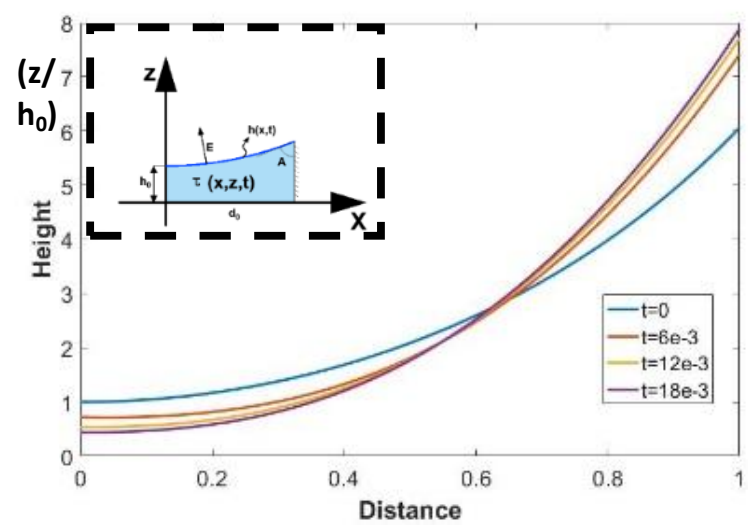

(b)

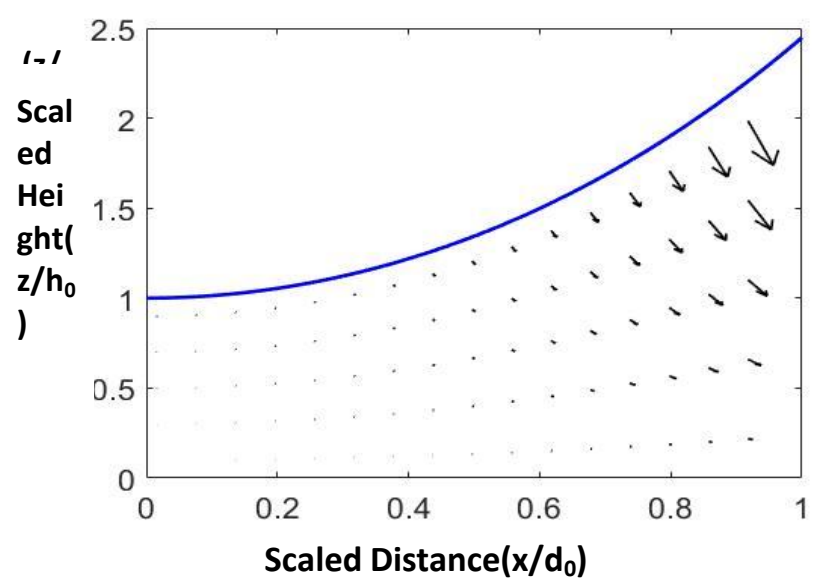

Eq. 27

Scal 'ith the above boundary condition, the ed ace evolution could be numerically Heig Jlifying to an ordinary differential equation $\mathbf{h t}(\mathbf{z}$ । finite difference approximation. Then, it is $/ h_{0}$ ) ulated by ODE $15 \mathrm{~s}$ provided by MATLAB. The vative uses the centred difference roximation for the continuous parts and -sided difference for the endpoints.

.... surface evolution of the top-right part of the capillary bridge is presented in the

Figure 3(a). Scaled Distance $\left(\mathbf{x} / \mathbf{d}_{0}\right)$ it the top-right part ce as shown in the schematic diagram in the dashed box, and are shown in drying time course. 
Figure 3: (a) Surface evolution of the capillary bridge surface with pure water. The coloured curve represents the top-right part of the capillary bridge surface as shown in the schematic diagram in the dashed box. The mass transfer coefficient $\left(k_{p}\right)$ used is 1e-3 with scaled time interval of 6e-3. (b) The velocity profile during desiccation. The direction and length of arrow indicate the inner flow trend and magnitude. The distance and height presented in the figures are all scaled.

Generally, the surface shape of the capillary bridge with pure water is not significantly transformed, still maintaining the curved shape. The surface height around $x=0$ is reduced slowly while the height at the end section is even increased in the drying condition.

This could be easily explained by the inner flow velocity profile(

Figure 3(b)). Since there is no solute concentration gradient, the latter part of the inner flow velocity is removed (Eq. 28). In other words, Marangoni Effect is not involved in this scenario. The velocity is governed by Capillary Effect only (Eq.28). The magnitude and direction of velocity are only dependent on the surface height and third derivative. The third derivative

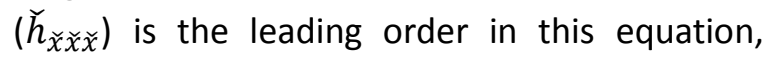
which increases from $\breve{x}=0$ to $\breve{x}=1$. Therefore, the length of arrow in

Figure $3(\mathrm{~b})$ indicates magnitude of velocity increases from $\breve{x}=0$ to $\breve{x}=1$.

$$
\bar{u}=\frac{1}{\breve{\mu}(\check{\tau})} \frac{\epsilon^{3}}{C a} \frac{\breve{h}^{2}}{3} \cdot \check{h}_{\check{x} \check{x} \check{x}}
$$

Eq. 28

Based on the velocity profile, it can clearly be seen that liquid is pushed from $\breve{x}=0$ to the end $\check{x}=11$

Figure $3(b))$, which results in the upsurge of surface height at the sides $(\check{x}=1)$ even in the desiccation environment.
At central point $(\check{x}=0)$, the curvature of the surface equals to zero, leading to third derivative of height $\left(\breve{h}_{\breve{x} \check{x} \check{x}}\right)$ in Eq. 28 is equal to zero. It means the surface height of the central point $(\breve{x}=0)$ is only governed by the evaporation rate.

However, owing to the curvature, the region next to the central point generates the capillary flow towards the side when evaporation commences and consequently reduces the surface height. Then, the area near the centre of the liquid bridge $(\breve{x}=0)$ transforms from the initial curve to a flat line gradually. In the course of drying, the flattening of the surface spreads from the centre and continues until the capillary bridge rupture at the centre. This simulation results are in agreement with the experiment result from previous work ${ }^{32-35}$.

\subsection{Simulation results with different solute concentration}

Unlike the case for pure water, mathematical construction of the capillary bridge with a trehalose solution is much more complex. The boundary condition of no-flux flow through the plane of symmetry mentioned above (Eq. 27) is not sufficient to solve all the equation. The initially uniform trehalose concentration distribution and non-penetration substrate are applied.

$$
\frac{\check{\partial \tau}}{\partial \check{x}}(\check{x}=0, \check{t})=0
$$

$$
\check{\tau}(\check{x}, \check{t}=0)=\text { constant }
$$

\subsubsection{Initial Trehalose Concentration=1M}

After relating all the conditions above into the numerical solution, the evolution of surface height and bulk concentration of the capillary bridge through the drying process are calculated (Figure 4). The coloured curves represent the top-right part of the capillary bridge surface.

The initial capillary bridge curve (Figure 4 (a)) is flattened when drying commences (Figure 4 
(a-c)). After a $12 \mathrm{e}-5$ scaled time period, liquid accumulates at the centre point (Figure 4 (d)), which is completely opposite to the situation with the pure water capillary bridge.

This phenomenon is driven by the trehalose concentration gradient along the surface (Figure 4 (e)). At the start, trehalose concentration is identical, leading to same drying rate. Owing to different volumes at different points along the concave surface, the changing rate of concentration with the respect of time varies along the $X$-axis (Figure4 (f)), decreasing from $\check{x}=0$. Then, the concentration of trehalose at the centre $(\check{x}=0)$, the thinnest part, would be the greatest and a concentration gradient along the surface is thus established

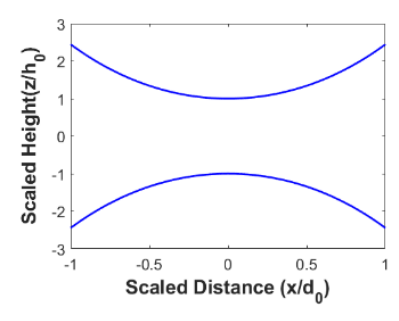

(a) $\check{t}=0$

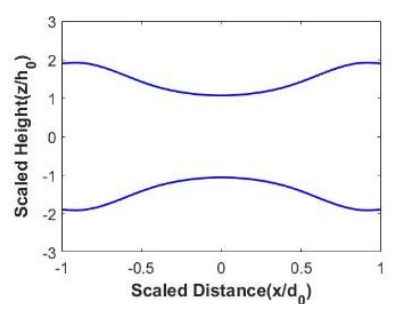

(b) $\check{t}=4 \mathrm{e}-5$

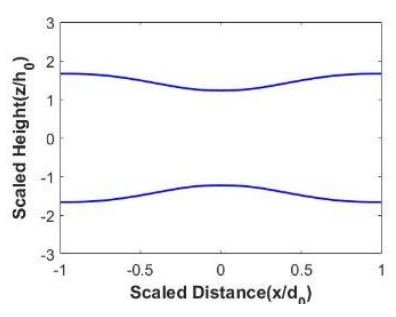

(c) $\check{t}=8 \mathrm{e}-5$

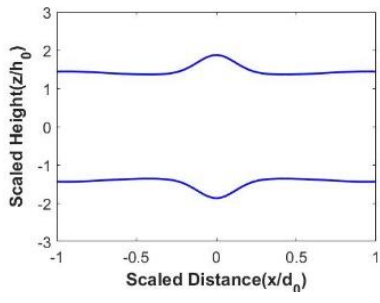

(d) $\check{t}=12 \mathrm{e}-5$

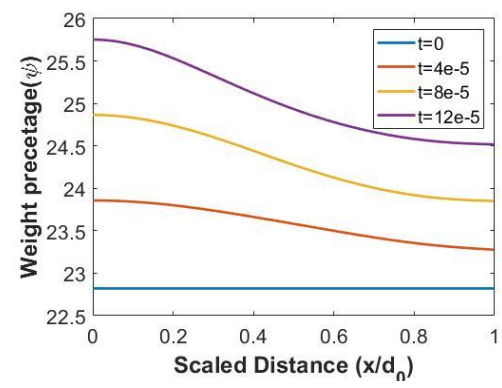

(e)

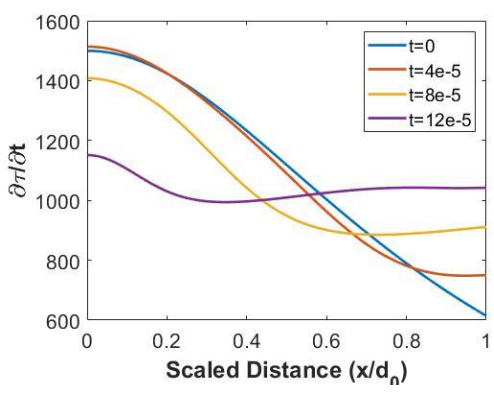

(f)

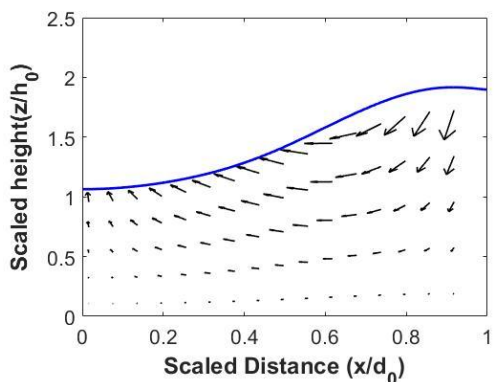

(g)

Figure 4: The evolution of capillary bridge surface with the Marangoni effect with $1 M$ initial solution. The mass transfer coefficient $\left(k_{p}\right)$ used is $1 e-3$ with a scaled time interval of $4 e-5$. (a-d) Evolution of surface height over scaled time, (e) Evolution of trehalose concentration ( $f$ ) Changing rate of concentration with the respect of time $(\overline{\partial \tau} / \overline{\partial t})$, (g)Velocity profile of inner flow at the second time interval(t=4e-5). The direction and length of arrow indicate the inner flow direction and magnitude. The distance and height presented in the figures are all scaled

Due to the properties of trehalose, the increasing concentration would increase the surface tension of the solution. Liquid in the area of low surface tension is pulled to an area of high surface tension by the Marangoni effect (Figure 4 (g)).

The thickness of the central area is increased by Marangoni flow and the concentration at that point is still the highest point across the bridge. In the course of drying, high concentration trehalose increases the viscosity of the capillary bridge and halt the inner flow. In the meantime, high sugar concentration also could impede the drying according to the Raoult's law. Therefore, at the end of evaporation, the solute, trehalose, will be gathered and vitrified at the central area, which prevents bridge rupture.

\subsubsection{Initial Trehalose Concentration=0.5M}

For low initial trehalose concentration $(0.5 \mathrm{M})$, the evolution of the right-top surface height has been presented in Figure 5(a). Up to $\check{t}=8 \mathrm{e}-5$, the tendency could be similar as the 
situation in $1 \mathrm{M}$ trehalose drying. Liquid accumulates at the centre due to the Marangoni flow. However, the volume of centre, increased by Marangoni flow, hinders the accumulation of trehalose at the central area shown in Figure 5(b).

At some point after $\breve{t}=8 \mathrm{e}-5$, the concentration at centre, or the first point, volume of which is compensated by the Marangoni flow, is the same as the concentration of the point next to the centre, the second point. Owing to the concentrations being equal, the drying rates during next time interval are identical. Since the volume of first point is greater than the second point, the concentration of second point is greatest in the next time interval. Another Marangoni flow is driven from the centre $(\breve{x}=0)$ to the second point. 


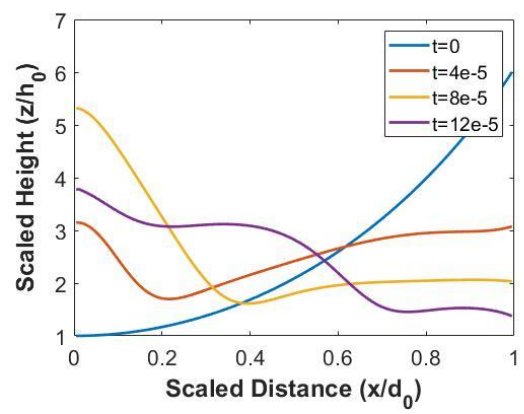

(a)

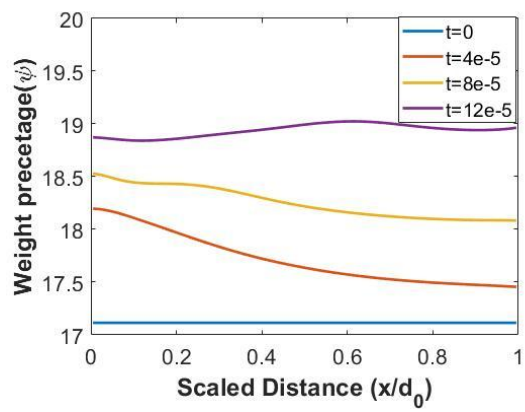

(b)

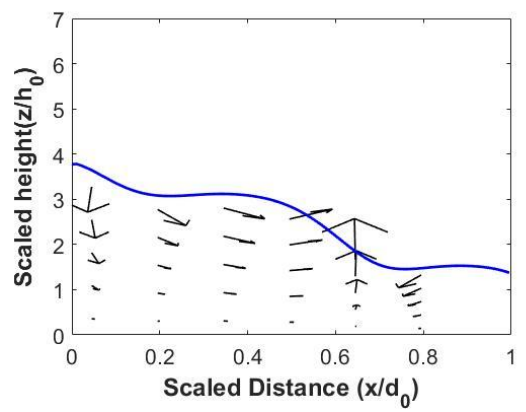

(c)

Figure 5: (a) The evolution of the right-top surface height of capillary bridge with $0.5 \mathrm{M}$ initial concentration. The coloured curve denotes the surface height of the capillary bridge. The legend was attached. (b) trehalose concentration along the surface during the drying. The mass transfer coefficient $\left(k_{p}\right)$ used is 1e-3 with scaled time interval of $4 e-5$. Different coloured curves indicate the surface height at different time slot. The legend was attached. (c) The velocity profile of inner flow at $\check{t}=12 e-5$. The distance and height presented in the figures are all scaled.

At some point afterwards, the same situation will occur between the second point and third point. This 'chain reaction' mechanism pushes liquid away from the central area, which is clearly seen from the velocity profile at $t=12 e-5$ shown in Figure 5(c). At the point with the greatest concentration of trehalose, liquid is gathered from the both sides.

Depending on the drying rate, there are two possible conformations of the final state. In the first condition, the glassy state of trehalose is formed somewhere between the centre $(\check{x}=0)$ and the end $(\check{x}=1)$. Then, the glassy matrix spreads from that point to the whole capillary bridge. If the drying rate was rather small, the reverse Marangoni flow would flatten the capillary bridge and form a glassy matrix at the edge ( $\check{x}=1)$.

At lower sugar concentration $(<0.5 \mathrm{M})$, another situation could occur. The concentration gradient created by the drying at the start cannot initiate sufficient Marangoni flow to overcome capillary flow. The surface instability would be similar to a pure water capillary bridge. Liquid is pushed to the end $(\check{x}=1)$ and the capillary bridge may rupture in this scenario.

\subsubsection{Initial Trehalose Concentration=2M}

Using $2 \mathrm{M}$ trehalose solution, almost the saturation point of trehalose at room temperature, the surface evolution progresses more simply (Figure $6(\mathrm{a})$ ). The amorphous state with large viscosity, weight percentage over $70 \%$, rapidly appears at the centre (Figure 6 (b)) and spreads out to result in an even bridge structure.

However, this simulation above is over simplified because high concentration trehalose would form a skin layer at the surface rapidly, which has a very high viscosity. Thus, the assumption of rapid vertical diffusion is no longer valid. Also, this skin layer could significantly cut the drying rate to prolong the desiccation process significantly. The skin layer movement is related to the Stefan problem, which is not considered in this work. 

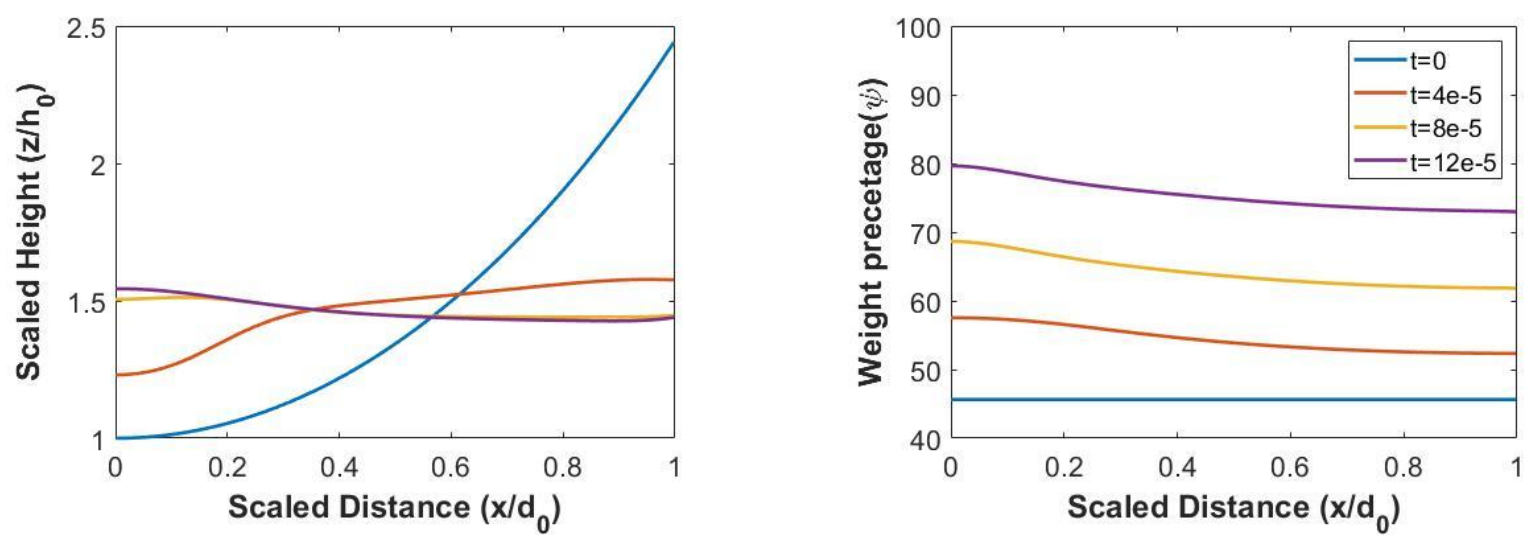

Figure 6: (a) The evolution of the right-top surface height of capillary bridge with $2 \mathrm{M}$ initial concentration. The coloured curves denote the surface height of the capillary bridge. (b) Trehalose concentration along the surface during the evaporation. The mass transfer coefficient $\left(k_{p}\right)$ used is $1 e-2$ with a scaled time interval of 4e-5. Different coloured curves mean the surface height at different time intervals. The distance and height presented in the figures are all scaled.

\section{Conclusions}

The wetting morphology of fibres can be predicted by the Capillary Bridge number (CB number), which is obtained from structure geometry. When $\mathrm{CB}$ number is greater than zero for given fibre matrix properties, a capillary bridge can be formed between fibres. However, the unknown nature of the relationship between principle radius and ambient environment limits the application of Capillary Bridge number but is related to the volume.

The surface instability of the capillary bridge formed form pure water or sugar solution of different concentration was simulated numerically using MATLAB. In the pure water situation, liquid is driven from the centre $(\check{x}=0)$ to the end $(\check{x}=1)$ by capillary pressure, which leads to the surface of the central area become flat. Then, the flatness is retained until capillary bridge rupture due to evaporation. The simulation result is in agreement with the experimental observations based on the previous work $^{32-35}$.
In a sugar solution-loaded capillary bridge, Marangoni flow is introduced by a surface tension gradient. Marangoni flow overcomes the capillary flow and pushes liquid towards the centre $(\check{x}=0)$ regardless of the sugar concentrations. The thickness of the central area $(\check{x}=0)$ is increased by Marangoni flow. The drying rate reduces with the increase of trehalose concentration and is stopped by the sugar skin formed on the surface. Therefore, a trehalose-loaded capillary bridge will not rupture except at extremely low initial trehalose concentration with a relatively high drying rate.

This model also can be extended to any solution, where there are known relationships between solute concentration and viscosity, diffusion coefficient, and surface tension. Also, the rate of drying influences the outcome through the rate of changing concentration during the drying process. It is quite similar to changing the initial concentration.

The modelling and simulation results revealed the complexity of capillary bridge formation for trehalose-fibre combination and its dynamics during drying. Giving a set of physical, thermodynamic and transport properties, it is possible to predict whether the 
capillary bridges can be form and whether they will not break during drying, which are important to the MART drying of biological therapeutics.

\section{Acknowledgements}

This work is partially supported by China Regenerative Medicine International Limited (CRMI), Hong Kong. Yejiong Yu would like to show his gratitude to Catriona Inverarity for her critical reading and suggestions.

\section{References}

1. Alcock, R. et al. Long-Term Thermostabilization of Live Poxviral and Adenoviral Vaccine Vectors at Supraphysiological Temperatures in Carbohydrate Glass. Vaccine 2, 1-8 (2010).

2. Dulal, P. et al. Potency of a thermostabilised chimpanzee adenovirus Rift Valley Fever vaccine in cattle. Vaccine 34, 2296-2298 (2016).

3. Dulal, P. Investigation of sugar-membrane vaccine stabilisation for improved vaccine thermostability and delivery (PhD thesis). (University of Oxford., 2016).

4. Majewski, R. L. et al. Bioencapsulation Technologies in Tissue Engineering. J. Appl. Biomater. Funct. Mater. 14, 395-403 (2016).

5. Fan, W.-X., Ma, X.-H., Ge, D., Liu, T.-Q. \& Cui, Z.-F. Cryoprotectants for the vitrification of corneal endothelial cells. Cryobiology 58, 28-36 (2009).

6. Ma, X., Sun, B.-B., Liu, T.-Q. \& Cui, Z.-F. Study of microscopic experiment for freezing/vitrifying characteristics of CPA solution and cell suspension. Dalian Ligong Daxue Xuebao/Journal Dalian Univ. Technol. 45, 517-521 (2005).

7. Kralchevsky, P. \& Nagayama, K. Capillary Bridges and Capillary-Bridge Forces. in Studies in Interface Science 10, 469-502 (2001).

8. Bostwick, J. B. \& Steen, P. H. Stability of Constrained Capillary Surfaces. Annu. Rev. 47:539-68(2015).

9. Willett, C. D., Adams, M. J., Johnson, S. A. \& Seville, J. P. K. Capillary Bridges between Two Spherical Bodies. 9396-9405 (2000). doi:10.1021/la000657y

10. Guo, Y. \& Curtis, J. Discrete Element Method Simulations for Complex Granular Flows. Annu. Rev. Fluid Mech. 47, 21-46 (2015).

11. Anand, A., Curtis, J. S., Wassgren, C. R., Hancock, B. C. \& Ketterhagen, W. R. Predicting discharge dynamics of wet cohesive particles from a rectangular hopper using the discrete element method (DEM). Chem. Eng. Sci. 64, 5268-5275
(2009)

12. Gao, S., Jin, L., Du, J. \& Liu, H. The Liquid-Bridge with Large Gap in Micro Structural Systems. 2011, 404-415 (2011).

13. Curran, K., Colin, S., Baldas, L. \& Davies, M. Liquid bridge instability applied to microfluidics. Microfluid. Nanofluidics 1, 336-345 (2005).

14. Morrow, N. R. Wettability and Its Effect on Oil Recovery. Society of Petroleum Engineers,14761477 (1990).

15. $\mathrm{Xu}, \mathrm{J}$. et al. Self-Assembly of Gradient Concentric Rings via Solvent Evaporation from a Capillary Bridge. Phys. Rev. Lett. 96, 66104 (2006).

16. Thomas, Y. An essay on the cohesion of fluids. Phil. Trans. R. Soc. 95, (1805).

17. Dörmann, M. \& Schmid, H. Simulation of Capillary Bridges between Particles. Procedia Eng. 102, 1423 (2015).

18. Wang, L. \& McCarthy, T. J. Capillary-bridge derived particles with negative Gaussian curvature. Proc. Natl. Acad. Sci. 112, 2664-2669 (2015).

19. Anjali, T. G. \& Basavaraj, M. G. Shape-Induced Deformation, Capillary Bridging, and SelfAssembly of Cuboids at the Fluid - Fluid Interface. (2017). doi:10.1021/acs.langmuir.6b03866

20. Bezdenejnykh, N. A., Meseguer, J., Perales, J. M., Aerodinamica, L. De \& Introduction, I. Experimental analysis of stability limits of capillary liquid bridges. i, (1991).

21. Chen, T.-Y., Tsamopoulos, J. A. \& Good, R. J. Capillary bridges between parallel and nonparallel surfaces and their stability. J. Colloid Interface Sci. 151, 49-69 (1992).

22. Bostwick, J. B. \& Steen, P. H. Stability of Constrained Capillary Surfaces. Annu. Rev. Fluid Mech. 47, 539-568 (2015).

23. Bezdenejnykh, N. A., Meseguer, J. \& Perales, J. M. Experimental analysis of stability limits of capillary liquid bridges. Phys. of Fluid A, 677, (2011).

24. Ruiter, R. De et al. Stability Limits of Capillary Bridges: How Contact Angle Hysteresis Affects Morphology Transitions of Liquid Microstructures. Phys. Rev. Lett. 114, 234501, 1-5 (2015).

25. BJ. Lowry and PH steen. Capillary surfaces: stability from families of equilibria with application to the liquid bridge. Proceedings: Mathematical and Physical Science, Vol 449, No 1937, 411-439 (1995).

26. Cooray, H., Huppert, H. E. \& Neufeld, J. A. Maximal liquid bridges between horizontal cylinders. 472. Proc. R. Soc A(2016).

27. Protiere, S., Duprat, C. \& Stone, H. A. Wetting on two parallel fi bers: drop to column transitions. Soft Matter m, 271-276 (2013).

28. Virozub, A., Haimovich, N. \& Brandon, S. ThreeDimensional Simulations of Liquid Bridges between Two Cylinders: Forces, Energies, and 
Torques. Langmuir 88, 12837-12842 (2009).

29. Beebe, A. Y. \& Stone, H. A. Wetting of flexible fibre arrays `. Nature 5-8 (2012).

30. Boulogne, F., Sauret, A., Soh, B., Dressaire, E. \& Stone, H. A. Mechanical Tuning of the Evaporation Rate of Liquid on Crossed Fibers. Langmuir 31, 3094-3100 (2015).

31. Cho, K. et al. Low internal pressure in femtoliter water capillary bridges reduces evaporation rates. Nat. Publ. Gr. 1-7 (2016). doi:10.1038/srep22232

32. Mielniczuk, B. \& Hueckel, T. Rupture of an Evaporating Liquid Bridge between Two Grains. Acta Geophys. (2014). doi:10.2478/s11600-0140225-6

33. Mielniczuk, B. et al. Effect of water on granular matter mechanics, local scale : evaporation, extension and rupture of liquid bridges To cite this version : HAL Id : hal-01102747 (2015)33..

34. Mastrangeli, M. The Fluid Joint : The Soft Spot of Micro- and Nanosystems. (2017).

doi:10.1002/adma.201501260

35. Portuguez, E., Alzina, A., Michaud, P. \& Smith, A. Evaporation Kinetics and Breaking of a Thin Water Liquid Bridge between Two Plates of Silicon Wafer. 157-166 (2016).

36. Aziz, H. \& Tafreshi, H. V. Competing forces on a liquid bridge between parallel and orthogonal dissimilar fibers. Soft Matter 15, 6967-6977 (2019).

37. Cheong, B. H.-P., Lye, J. K. K., Backhous, S., Liew, O. W. \& Ng, T. W. Microplates based on liquid bridges between glass rods. J. Colloid Interface Sci. 397, 177-184 (2013).

38. Princen, H. M. Capillary phenomena in assemblies of parallel cylinders: III. Liquid Columns between Horizontal Parallel Cylinders. J. Colloid Interface Sci. 34, 171-184 (1970).

39. Neeson, M. J., Dagastine, R. R., Chan, Y. C. \& Tabor, R. F. Evaporation of a capillary bridge between a particle and a surfacet. Soft Matter, 42 8489-8499 (2014). doi:10.1039/c4sm01826e

40. Kralchevsky, P. \& Nagayama, K. Capillary Bridges and Capillary-Bridge Forces. Book: Particles at Fluid interfaces and Membranes, 469-502 (2001).

41. Do, M. \& Schmid, H. Simulation of Capillary Bridges between Nanoscale Particles. Langmuir 2014, 30, 4, 1055-1062 (2014). doi:10.1021/la404409k

42. Rynhart, P. R., Mclachlan, R., Jones, J. R. \& Mckibbin, R. Solution of the Young-Laplace equation for three particles. Res. Lett. Inf. Sci 5, 119-127 (2003).

43. Nguyen, H. N. G., Millet, O. \& Gagneux, G. On the capillary bridge between spherical particles of unequal size: analytical and experimental approaches. Contin. Mech. Thermodyn. 31, 225237 (2019)
44. Company, L. B., Division, D. \& Jer, N. Capillary Phenomena in Assemblies of Parallel Cylinders III . Liquid Columns between Horizontal Parallel Cylinders. Journal of Colloid and interface sicence, 34,2, 171-184 (1970).

45. Yang, L., Tu, Y. \& Fang, H. Modeling the rupture of a capillary liquid bridge between a sphere and plane. Soft Matter 6, 6178-6182 (2010).

46. De Lazzer, A., Dreyer, M. \& Rath, H. J. Particle Surface capillary forces. Langmuir 15, 4551-4559 (1999).

47. Van Honschoten, J. W., Brunets, N. \& Tas, N. R. Capillarity at the nanoscale. Chem. Soc. Rev. 39, 1096-1114 (2010).

48. Weeks, B. L., Vaughn, M. W. \& Deyoreo, J. J. Direct imaging of meniscus formation in atomic force microscopy using environmental scanning electron microscopy. Langmuir 21, 8096-8098 (2005).

49. Kim, S., Kim, D., Kim, J., An, S. \& Jhe, W. Direct Evidence for Curvature-Dependent Surface Tension in Capillary Condensation: Kelvin Equation at Molecular Scale. Phys. Rev. X 8, (2018).

50. Craster, R. V, Matar, O. K. \& Sefiane, K. Pinning, Retraction, and Terracing of Evaporating Droplets Containing Nanoparticles. Langmuir 25, 36013609 (2009).

51. Karapetsas, G., Sahu, K. C. \& Matar, O. K. Evaporation of Sessile Droplets Laden with Particles and Insoluble Surfactants. Langmuir 32, 6871-6881 (2016).

52. Beattie, G. M. et al. Trehalose: a cryoprotectant that enhances recovery and preserves function of human pancreatic islets after long-term storage. Diabetes 46, 519-523 (1997).

53. Martinetti, D. et al. Effect of trehalose on cryopreservation of pure peripheral blood stem cells. Biomed. reports 6, 314-318 (2017).

54. Rodrigues, J. P. et al. Evaluation of trehalose and sucrose as cryoprotectants for hematopoietic stem cells of umbilical cord blood. Cryobiology 56, 144-151 (2008).

55. Osman, A., Shahidzadeh, N., Stitt, H. \& Shokri, N. Morphological transformations during drying of surfactant-nanofluid droplets. J. Ind. Eng. Chem. 67, 92-98 (2018).

56. Trybala, A. Evaporation of sessile droplets Evaporation of sessile droplets.figshare. Jurnal contribution. https://hdl.handle.net/2134/25771 (2017).

57. Galmarini, M. V., Baeza, R., Sanchez, V., Zamora, M. C. \& Chirife, J. Comparison of the viscosity of trehalose and sucrose solutions at various temperatures: Effect of guar gum addition. LWT Food Sci. Technol. 44, 186-190 (2011).

58. Colaço, C. A. L. S. \& Roser, B. Trehalose-a multifunctional additive for food preservation. in 
Food Packaging and Preservation (ed. Mathlouthi, M.) 123-140 (Springer US, 1994).

doi:10.1007/978-1-4615-2173-0_7

59. Grasmeijer, N., Frijlink, H. W. \& Hinrichs, W. L. J. Model to predict inhomogeneous protein-sugar distribution in powders prepared by spray drying. J. Aerosol Sci. 101, 22-33 (2016).

60. Brutin, D. Recent advances in droplet wetting and evaporation. Chem Soc Rev 558-585 (2018). doi:10.1039/c6cs00902f

61. Bhardwaj, R., Fang, X. \& Attinger, D. Pattern formation during the evaporation of a colloidal nanoliter drop: a numerical and experimental study. New J. Phys. 11, 75020 (2009).

62. Kita, Y., Arakawa, T., Lin, T. yin \& Timasheff, S. N. Contribution of the Surface Free Energy Perturbation to Protein-Solvent Interactions. Biochemistry 33, 15178-15189 (1994).

63. Jensen, O. E. \& Grotberg, J. B. The spreading of heat or soluble surfactant along a thin liquid film The spreading of heat or solluble surfactant along a thin liquid film. Phys. of Fluid A,5,58, (1993). 


\section{Appendix A: Formulation Details of Drying Dynamic Simulation}

The governing equations and boundary conditions are non-dimensionlized consistent with following scalings. The tildes represent the dimensionless variables.

$$
\begin{array}{ccc}
x=d_{0} \check{x} & z=h_{0} \check{z} & u=U_{0} \cdot \check{u} \\
h=h_{0} \check{h} & \tau=\tau_{i} \check{\tau} & w=\frac{h_{0}}{d_{0}} U_{0} \cdot \check{u} \\
\mu=\mu_{0} \check{\mu} & D=D_{0} \check{D} & t=\frac{d_{0}}{U_{0}} \check{t}
\end{array}
$$

$\mu_{0}$ and $D_{0}$ are the initial viscosity and diffusion coefficient based on the initial concentration. The velocity scale is based on the balance of capillary force and viscous.

$$
U_{0}=\frac{h_{0}{ }^{3}}{\mu_{0} d_{0}{ }^{3}} \sqrt{\sigma_{0}}
$$

Thus, the continuity and momentum conservation equations are scaled to:

$$
\check{u}_{\check{x}}+\breve{w}_{\check{z}}=0
$$

$$
\begin{aligned}
& \operatorname{Re} \cdot \epsilon\left(\check{u} \cdot \check{u}_{\check{x}}+\check{w} \cdot \check{u}_{\breve{z}}\right) \\
& =-\check{p}_{\check{x}}+\check{\mu}(\check{\tau}) \cdot \epsilon^{2} \check{u}_{\breve{x} \check{x}}+\check{\mu}(\check{\tau}) \check{u}_{\check{z} \check{z}}
\end{aligned}
$$

$$
\begin{gathered}
R e \cdot \epsilon^{3}\left(\check{u} \cdot \breve{w}_{\check{x}}+\breve{w} \cdot \breve{w}_{\check{z}}\right)= \\
-\check{p}_{\check{z}}+\epsilon^{2} \breve{\mu}(\check{\tau})\left(\epsilon^{2} \breve{w}_{\check{x} \check{x}}+\breve{w}_{\check{z} \check{z}}\right)
\end{gathered}
$$

where,

$$
R e=\frac{\rho U_{0} D}{\mu_{0}}, \epsilon=h_{0} / d_{0}
$$

With the boundary condition at the substrate and interface, respectively.

$$
\breve{u}=\breve{w}=0 \text {, at } z=0
$$

The boundary conditions at the interface is nondimensionalised.

Normal stress balance:

$$
\begin{aligned}
\frac{2 \epsilon^{2}}{\Omega^{2}}\left[\epsilon^{2} \breve{u}_{\breve{x}}\left(\breve{h}_{\breve{x}}\right)^{2}\right. & \left.-\breve{h}_{\check{x}}\left(\check{u}_{\breve{z}}+\epsilon^{2} \breve{w}_{\check{x}}\right)+\breve{w}_{\check{z}}\right]-p \\
& =\frac{\epsilon^{3}}{C a} \cdot \breve{h}_{\breve{x} \breve{x}}
\end{aligned}
$$

where

$$
\Omega=\sqrt{1+\left(\epsilon \breve{h}_{\check{x}}\right)^{2}}
$$

$$
\text { Ca }(\text { Capillary number })=\frac{\mu_{0} U_{0}}{\sigma}
$$

Tangential stress balance:

$$
-2 \epsilon^{2} \breve{h}_{\check{x}} \check{u}_{\check{x}}-\breve{u}_{\check{z}}+\epsilon^{2} \breve{w}_{\check{x}}-\epsilon^{2}\left(\breve{h}_{\check{x}}\right)^{2}\left(\breve{u}_{\check{z}}+\epsilon^{2} \breve{w}_{\check{x}}\right)
$$

$$
+2 \epsilon^{2} \breve{h}_{\check{x}} \breve{w}_{\check{z}}=\frac{M}{p e}\left[\check{\tau}_{\check{x}}+\check{h}_{\breve{x}} \check{\tau}_{\breve{z}}\right]
$$

where

$$
M(\text { Marangoni number })=\frac{\gamma \tau_{0} h_{0}}{\mu_{0} \cdot D}
$$

Kinematic boundary condition

$$
E=\frac{d_{0}}{h_{0} U_{0}}\left(\check{w}-\check{u} \cdot \frac{\partial \breve{h}}{\partial \check{x}}-\frac{\partial \breve{h}}{\partial \check{t}}\right)
$$

The evolution of trehalose mass fraction is governed by the dimensionless convective diffusion equation,

$$
\check{\tau}_{\check{t}}+\check{u} \cdot \check{\tau}_{\check{x}}+\check{w} \cdot \check{\tau}_{\check{z}}=\frac{1}{P e \epsilon^{2}}\left(\epsilon^{2} \cdot \check{\tau}_{\check{x} \check{x}}+\check{\tau}_{\check{z} \check{z}}\right)
$$

with the boundary condition at substrate and interface, respectively.

$$
\frac{\partial \check{\tau}}{\partial \check{z}}_{\mid z=0}=0
$$

$$
\begin{aligned}
\epsilon^{2} P e \cdot \check{\tau} \cdot\left(\check{w}-\check{u} \cdot \frac{\partial \check{h}}{\partial \check{x}}-\frac{\partial \check{h}}{\partial \check{t}}\right) \\
=-\epsilon^{2} \breve{h}_{x} \check{\tau}_{x}+\check{\tau}_{z}
\end{aligned}
$$

Peclet number $\left(P e=\frac{U_{0} d_{0}}{D}\right)$ is a parameter to evaluate the prioritization of convective against diffusive transportation during the desiccation. In this work, rapid vertical diffusion is imposed. Under this correspondence, the mass fraction could be expanded as following ${ }^{50,62}$ :

$$
\check{\tau}(x, z, t)=\check{\tau}_{0}(\check{x}, \check{t})+\epsilon^{2} P e \cdot \check{\tau}_{1}(\check{x}, \check{z}, \check{t})
$$

Then, the convective diffusion equation ( $A .19)$ is substituted as

$$
\begin{gathered}
\check{\tau}_{0 \check{t}}+\epsilon^{2} P e \cdot \check{\tau}_{1_{t}}+\check{u} \cdot\left(\check{\tau}_{0 \check{x}}+\epsilon^{2} P e \cdot \check{\tau}_{1 \check{x}}\right) \\
+\check{w} \cdot \epsilon^{2} P e \cdot \check{\tau}_{1_{\check{z}}}=\frac{1}{P e} \check{\tau}_{0 \check{x} \check{x}}+\check{\tau}_{1 \check{z} \check{z}}
\end{gathered}
$$

with the boundary conditions

$$
\begin{gathered}
\frac{\partial \check{\mathrm{t}}_{1}}{\partial \check{\mathrm{z}}}=0 \text { at } z=0 \\
\left(\check{\tau}_{0}+\epsilon^{2} P e \cdot \check{\tau}_{1}\right) \cdot\left(\check{w}-\check{u} \cdot \frac{\partial \check{h}}{\partial \check{x}}-\frac{\partial \check{h}}{\partial \check{t}}\right)^{A .13} \\
=\frac{-\check{h}_{x} \cdot\left(\check{\tau}_{0_{x}}+\epsilon^{2} P e \cdot \check{\tau}_{1 \check{x}}\right)}{P e}+\check{\tau}_{1 \breve{z}}, \text { at } z=h
\end{gathered}
$$


Owing to $\epsilon=h_{0} / d_{0} \ll 1$, the lubrication approximation is applied to simplify the system to the leading orders of all above dimensionless equations. The leading order of continuity, momentum conservation and trehalose diffusion equations are yielded.

$$
\begin{gathered}
\check{u}_{\check{x}}+\breve{w}_{\check{z}}=0 \\
\check{p}_{\check{x}}=\check{\mu}(\check{\tau}) \cdot \check{u}_{\check{z} \check{z}} \\
\check{p}_{\check{z}}=0
\end{gathered}
$$

From the $x$-component of Navier-Stokes equation (A. 16), the horizontal velocity is mainly dependent with the pressure. which is not varied along the vertical direction (A. 17). Hence, to solve this second derivate momentum equation, two boundary conditions are applied, no-slip wall condition (A. 18) and scaled tangential stress balance (A. 19).

$$
\begin{gathered}
\check{u}=\check{w}=0 \\
\check{\mu}(\check{\tau}) \frac{\partial \check{u}}{\partial \check{z}}=\frac{M a \cdot \breve{D}}{P e}\left(\frac{\partial \check{\tau}}{\partial \check{x}}+\frac{\partial \check{\mathrm{h}}}{\partial \check{x}} \cdot \frac{\partial \check{\tau}}{\partial \check{z}}\right)
\end{gathered}
$$

where $\mathrm{Ma}($ Marangoni number $)=\frac{\gamma \tau_{0} h_{0}}{\mu_{0} \cdot D_{0}}$

After solving the momentum conservation equation, the horizontal velocity profile was expressed as:

$$
\begin{array}{r}
\check{u}=\frac{1}{\check{\mu}(\check{\tau})}\left[\check{p_{\check{x}}}\left(\frac{\check{z}^{2}}{2}-\check{h} \check{z}\right)+\frac{M a \cdot \breve{D}}{P e}\right. \\
\left.\cdot\left(\frac{\partial \check{\tau}}{\partial \check{x}}+\frac{\partial \check{\mathrm{h}}}{\partial \check{x}} \cdot \frac{\partial \check{\tau}}{\partial \check{z}}\right) \check{z}\right]
\end{array}
$$

According to the simplified normal stress balance (A. 21), the pressure could be substituted, and the velocity was reviewed as the function of surface and height and local concentration (A. 22).

$$
-\check{p}=\frac{\epsilon^{3}}{C a} \cdot \check{h}_{\check{x} \check{x}}
$$

where,

$$
\text { Ca }(\text { Capillary number })=\frac{\mu_{0} U_{0}}{\sigma}
$$

$$
\begin{aligned}
\check{u}(\breve{h}, \check{\tau})=\frac{1}{\check{\mu}(\check{\tau})}[ & \frac{\epsilon^{3}}{C a} \cdot \breve{h}_{\breve{x} \check{x} \check{x}}\left(\frac{\check{z}^{2}}{2}-\check{h} \check{z}\right) \\
& \left.+\frac{M a \cdot \breve{D}}{P e} \cdot\left(\frac{\partial \check{\tau}}{\partial \check{x}}+\frac{\partial \check{\mathrm{h}}}{\partial \check{x}} \cdot \frac{\partial \check{\tau}}{\partial \check{z}}\right) \check{z}\right]
\end{aligned}
$$

Then, the convective diffusion equation was scaled as

$$
\check{\tau}_{0 \breve{t}}+\check{u} \cdot\left(\check{\tau}_{0 \check{x}}\right)=\frac{\breve{D}}{P e} \check{\tau}_{0} \check{x} \check{x}+\breve{D} \cdot \check{\tau}_{1 \check{z} \check{z}}
$$

with boundary conditions:

at the substrate, $z=0$

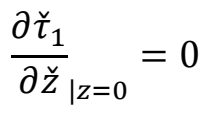

at the interface, $z=h$

$$
\frac{E}{\epsilon \cdot \check{D} U_{0}} \cdot \check{\tau}_{0}=\frac{-\check{h}_{x} \cdot \check{\tau}_{0 \check{x}}}{P e}+\check{\tau}_{1_{\check{z}}}
$$

To get the dynamic equation of Trehalose concentration liquid bridge, the crosssectional averaging technique was applied ${ }^{62}$. The diffusion equation (A. 23) could be reformed as:

$$
\widetilde{\tau_{0 \mathrm{t}}}+\overline{\bar{u}}{\widetilde{\tau_{0 \mathrm{x}}}}=\frac{\widetilde{\mathrm{D}}}{\mathrm{Pe}}{\widetilde{\tau_{0 \mathrm{x}}}}+\frac{1}{\check{h}}\left[\widetilde{\tau_{1_{z}}}\right]_{0}^{\breve{h}}
$$

where the last term could be extended with the boundary conditions A. 24 and A. 25. Thus, the governing equation of local concentration along the drying was stated as

$$
\frac{\partial \check{\tau}_{0}}{\partial t}=-\bar{u} \cdot \check{\tau}_{0 \check{x}}+\frac{\breve{D}}{P e \cdot \breve{h}}\left(\check{h} \check{\tau}_{0_{x}}\right)_{x}+\frac{\check{\tau}_{0}}{h U_{0} \epsilon} \cdot E
$$

The concentration distribution was used to simplify the velocity profile equation.

$$
\begin{aligned}
& \check{u}\left(\check{h}_{,} \breve{\tau}_{0}\right)=\frac{1}{\check{\mu}(\check{\tau})}\left[\frac{\epsilon^{3}}{C a} \cdot \check{h}_{\breve{x} \check{x} \check{x}}\left(\frac{\check{z}^{2}}{2}-\check{h} \check{z}\right)\right. \\
& \left.+\frac{M a \cdot \breve{D}}{P e} \cdot\left(\frac{\partial \check{\tau}_{0}}{\partial \check{x}}\right) \check{z}\right]
\end{aligned}
$$

As the other objective of this work, to solve the evolution of surface height along the desiccation is also an essential part, which could be illustrated by kinematic boundary condition.

$$
E=\frac{d_{0}}{h_{0} U_{0}}\left(\check{w}-\check{u} \cdot \frac{\partial \check{h}}{\partial \check{x}}-\frac{\partial \check{h}}{\partial \check{t}}\right)
$$


The mean horizontal simplified the kinematic boundary condition to eliminate the vertical velocity component calculations by Leibniz's Rule.

$$
-\frac{\partial \check{h} \bar{u}}{\partial \check{x}}=\check{w}-\check{u} \cdot \frac{\partial \check{h}}{\partial \check{x}}
$$

Thus, the evolution of surface height could be substituted and expressed as

$$
\frac{\partial \check{h}}{\partial \check{t}}=-\frac{h_{0} U_{0}}{d_{0}} E-\frac{\partial(\check{h} \bar{u})}{\partial \check{x}}
$$

where,

$$
\bar{u}=\frac{1}{\check{\mu}(\check{\tau})}\left[\frac{\epsilon^{3}}{C a} \frac{\breve{h}^{2}}{3} \cdot \check{h}_{\breve{x} \breve{x} \breve{x}}+\frac{M a \cdot \breve{D}}{P e} \cdot\left(\frac{\partial \check{\tau}_{0}}{\partial \check{x}}\right) \frac{\check{h}}{2}\right]
$$

\section{Appendix B: Parameters determination}

The relationship between trehalose concentration and viscosity needs to be determined before the numerical solution.

\subsection{Method}

\section{Materials and Method}

D- (+)-Trehalose dehydrate (Sigma Aldrich, $\geq$ 99\%) has been dissolved in Milli-Q water to configure different weight percentage sugar solutions gravimetrically. The range of Trehalose concentrations was from $0 \%$ to $62 \%$. When over $40 \% \mathrm{wt}$, trehalose could not dissolve easily in water at room temperature. In that case, the solutions were prepared by heating and mixing in sealed glass vials.

\section{Viscosity}

Viscosities of Trehalose solutions were measured by optical Rheometer (DWS RheoLabs from LS Instruments). Firstly, all the solutions were mixed with $500 \mathrm{~nm}$ nanoparticles in $2 \%$ weight percentage, which was referred to the calculation provided by LS Instruments to ensure the turbidity index $(I)$ was located between 5 and 30 . Otherwise, the results were invalid. Then, each mixture was transferred into $2 \mathrm{~mm}$ cuvette and placed into the machine. The measuring temperature was set to $9{ }^{\circ} \mathrm{C}$ by the software on the computer, which is the wet bulb temperature of room temperature $\left(25^{\circ} \mathrm{C}\right)$ with $5 \%$ relative humidity. When the temperature became constant, software was run, and the results were recorded.

\subsection{Experiments Results}

The dynamic viscosities (in units $c p$ ) of several different weight percentage Trehalose solutions have been determined by Rheometer. In this work, the dilution of adding nanoparticles was neglected.

Those two emperical number could be determined by rearranging the Eq. 12

$$
\ln (\mu)=A_{1} \psi+\ln \left(A_{0}\right)
$$

Substituting all the data into the B. 1, the tendency equation could be obtained from Figure B1.

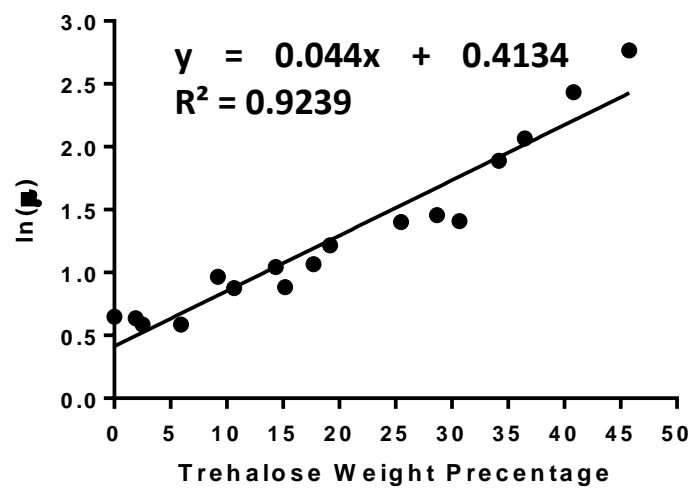

Figure B1: The calibriation of viscosities with different trehalose weight precentages. The slope and intercept are related with above two emperial parameters $A_{0}$ and $A_{1}$.

Two empirical numbers $A_{0}$ and $A_{1}$ are read based on tendency equation, which were $1.2932 \times 10^{-3}$ and 0.0471 respectively. However, the exponential approximation is valid when the trehalose weight lower than $50 \%$. If fitting the data over $50 \%$, the R Square number drops sharply, which considerably increased the error of approximation. 